workers, showed to be an effective and also measurable way to significantly reduce the level of WRS risk at workplace. Head Physicians and Head Nurses of EDs should consider that stress management programs aimed to improve work context factors associated with occupational stress are effective in minimising the impact of WRS on ED workers.

\section{THE COMMON SAFETY AND HEALTH HAZARDS AMONG LABORATORY WORKERS IN KALIRO AND IGANGA DISTRICTS, UGANDA}

Tom Eliatu-Occupational Laboratory Technician*. Ministry of Gender, Labour and Social Development, Department of Occupational Safety and Health, Kampala, Uganda

\subsection{6/oemed-2018-ICOHabstracts.929}

Introduction According World Health Organisation report 2006, health care facilities around the world employ over 59 million workers who are exposed to a complex variety of health and safety hazards during execution of their daily routine activities. This often exposes health care workers in developing Occupational diseases. Health-care workers (HCWs) need to be protected from these workplace hazards in order to have an adequate workforce of trained and healthy health personnel. The main objective of this study was to inspect and find out the common safety and health hazards among the health workers in the laboratories of the main hospitals in the above districts.

Methods This study was carried out using Uganda's Occupational Safety and Health check list which was administered to 33 respondents working in laboratories of the main Hospitals in the above districts. This study was conducted from August 2016 up to March 2017. The data was collected and analysed using Excel programme.

Results Out of the 33 respondents interviewed, 55\% were exposed to biological hazards, $45 \%$ had no previous exposure to it, $94 \%$ had exposure to physical hazards and $6 \%$ had no exposure to it, $6 \%$ had exposure to chemical hazards and 94\% had no previous exposure, no respondent had reported previous exposure to fire explosion and electrical hazards, $70 \%$ had experienced psychosocial hazards and 30\% had not experienced it and no respondent had experienced ergonomic hazards at this work places.

Discussion The findings from this study shows that psychosocial hazards is the most common safety and health hazards because of having a lot of work which often results to stress and violence. This is closely followed by biological hazard which is attributed to lack of proper personal protective equipment. No electrical hazard was reported due to remoteness of these areas and no ergonomic was also reported because respondents had no previous to exposure to lifting heavy object.

\section{CURRENT ISSUES FOR HEALTH CARE WORKERS}

Bonnie Rogers*. University of North Carolina, Chapel Hill, NC, USA

10.1136/oemed-2018-ICOHabstracts.930

Discuss Caring for Displaced Persons, Violence in Health Care Setting, health issues regarding nanotechnology, $\mathrm{OH}$ issues for Animal Handlers

Presenters: ${ }^{1}$ Rogers B, ${ }^{2}$ Iavicoli I, ${ }^{3}$ Nienhaus A
${ }^{1}$ University of North Carolina, Chapel Hill North Carolina, United States

${ }^{2}$ Section of Occupational Medicine, Department of Public Health, University of Naples Federico II, Naples, Italy

${ }^{3}$ University Clinics Hamburg Eppendorf Centre for Health Service Research in Nursing, Hamburg, Germany

\section{8a HEALTH HAZARDS IN VETERINARY CARE}

B Rogers. University of North Carolina, Chapel Hill, NC, USA

10.1136/oemed-2018-ICOHabstracts.931

Veterinary health care workers are exposed to a highly diverse set of hazards similar to human health care workers and include exposures to biological, chemical, enviro-mechanical, psychosocial, and physical agents. This session will provide a review of veterinary health care hazards. Biological hazards include exposures to infectious and zoonotic diseases, animal allergens, and biologic pharmaceuticals. Several biologic agent exposures such as tick-borne and Lyme diseases are of concern and transmission routes for many biological agents are not completely understood. Many personnel suffer from animal allergen exposure and assessments estimates as well as disease are needed. Chemical hazards similar to those in human health care such as disinfectant agents which are continuously used and have been associated with asthma, rhinitis, and contact dermatitis in healthcare workers, and antineoplastic drug exposure, primarily used to treat cancer in dogs and cats are of particular concern. Exposure estimates, hazard knowledge among veterinary personnel related to these agents, and current prevention practices including use of PPE are limited. Enviro-mechanical hazards include sharps risks, and ergonomic risks to unsafe equipment, heavy lifting of both animals and equipment, and awkward postures. With many more women in veterinary health care, reproductive risks are significant from physically demanding work, standing for prolonged periods of time, long working long hours, and chemical exposure. Physical hazards include animal-related injuries such as bites, scratches, and crushing injuries from large animals. Animal noise is a unique hazard in veterinary health care (particularly to dogs) and can contribute to hearing loss. Psychological hazards from work-related stress dealing with the care of sick and injured animals, euthanasia, and with human grief are prevalent requiring much more exploration. Safety culture, attitudes and beliefs around personal safety and the expectation that certain hazards are to be accepted and tolerated remains and must be examined and mitigated.

\section{8b NANOTECHNOLOGY IN MEDICAL FIELDS: POTENTIAL APPLICATIONS, TOXICOLOGICAL IMPLICATIONS, AND OCCUPATIONAL RISKS}

I lavicoli, V Leso. Section of Occupational Medicine, Department of Public Health, University of Naples Federico II, Naples, Italy

\subsection{6/oemed-2018-ICOHabstracts.932}

Introduction Advances in nanotechnologies over the last years supported the development of nano-sized applications for medical purposes due to their beneficial impact on detection, imaging and treatment of diseases. However, new human health hazards may emerge from nano-medicine, also for 